\title{
ONE- AND TWO-NODE HYBRID LEAKAGE-CORRECTED 3-D CMFD ALGORITHM FOR FAST AND ACCURATE PIN-WISE PWR ANALYSIS
}

\author{
Jaeha Kim, Hwanyeal Yu, and Yonghee Kim* \\ Department of Nuclear and Quantum Engineering, Korea Advance Institute of Science and \\ Technology, 291 Daehak-ro, Yueseong-gu, Daejeon, Korea, 34141, Republic of Korea \\ najaeha@kaist.ac.kr, hwanyealyu@kaist.ac.kr, yongheekim@kaist.ac.kr*
}

\begin{abstract}
The HCMFD (Hybrid Coarse-Mesh Finite Difference) with GPS (GET Plus SPH) method and its 3-D application are investigated in this paper. In the HCMFD algorithm, a parallel computing for a pin-by-pin core calculation can be performed very effectively with a nonlinear local-global iterative scheme. For an effective parallel computing, the one-node CMFD is used to solve a global eigenvalue problem. The conventional two-node CMFD is used for solving local fixed source problems with incoming current boundary conditions. The GPS method is a leakage correction method to correct the pin-wise XSs of the conventional GETbased two-step procedure. In the GPS method, the XS-dependent SPH factors are parameterized as a function of the pin-wise albedo information, current-to-flux ratio (CFR). With updated XS-wise SPH factors, the pin-wise XSs are corrected in order to improve the accuracy of the conventional two-step core analysis. The GPS method is implemented to an in-house pin-by-pin diffusion solver with the HCMFD algorithm to keep their strong points together. In this paper, several 3-D variant cores of KAIST-1A benchmark problem were chosen to demonstrate the combination of HCMFD and GPS (HCMFD-GPS) method.
\end{abstract}

KEYWORDS: Pin-by-pin diffusion analysis, HCMFD algorithm, GPS method, parallel computing.

\section{INTRODUCTION}

The assembly-wise homogenized group constants, the cross sections (XS) and discontinuity factors (DF), have an inevitable error due to the unrealistic reflective boundary condition in the lattice calculation. Such error is more significant to the pin-cell-homogenized group constants due to strong neighborhood effects. Meanwhile, the computing time for a conventional pin-by-pin diffusion analysis is not short enough to have any merit. These two demerits on both the accuracy and computing time are the reasons that the pin-by-pin diffusion analysis has not widely been used.

Recently, the authors devised a parallel computational scheme for a pin-by-pin nodal diffusion analysis, the Hybrid Coarse-Mesh Finite Difference (HCMFD) algorithm [1], and a new leakage correction method, called GET Plus SPH (GPS) method [2]. It has been shown that a whole-core pin-by-pin diffusion analysis can be performed in a very short time by the HCMFD algorithm, and also the feasibility of the GPS method in 2-D geometries has been demonstrated in the previous studies. By combining them, it is expected that a whole-core pin-by-pin diffusion analysis result with sufficient accuracy can be obtained within a very short time. 
In this work, the HCMFD algorithm and the GPS method are briefly introduced, and along with a combined code system, the feasibility of implementing GPS functions generated from the 2-D color-set calculations to several 3-D benchmark problems is assessed.

\section{PIN-BY-PIN DIFFUSION ANALYSIS METHODOLOGIES}

\subsection{HCMFD Algorithm}

In the HCMFD algorithm, two CMFD (Coarse-Mesh Finite Difference) methods are nonlinearly coupled with the nodal expansion method (NEM) applied on pin-level. To enable an efficient parallel computing, the whole domain is decomposed into subdomains, the fuel assemblies in a PWR. The global balance is governed by solving the global eigenvalue problem with the one-node CMFD method [3], represented by Eq. (1). Then the global balance information is transferred to each subdomain in terms of the boundary conditions, the fixed fission source and incoming partial currents on the boundary.

$$
\begin{aligned}
& A_{c m f d}^{\text {global }} \Phi_{\text {cmfd }}^{\text {global }}=\frac{1}{k} F_{c m f d}^{\text {global }} \Phi_{\text {cmfd }}^{\text {global }}, \\
& \Phi_{\text {cmfd }}^{\text {global }} \text { : global node-averaged fluxes, } \\
& A_{\text {cmfd }}^{\text {global }} \text { : global system matrix, } \\
& F_{\text {cmfd }}^{\text {global }} \text { : global fission source operator, } \\
& k \text { :multiplication factor. }
\end{aligned}
$$

The local fixed source problems defined in pin-wise heterogeneous fuel assemblies, represented by Eq. (2), are solved by the conventional two-node CMFD method [4] based on NEM, with the partial current boundary conditions. The problems are then homogenized for the following global calculation.

$$
\begin{gathered}
M_{i}^{\text {local }} \phi_{i}^{\text {local }}=S_{i}^{\text {local }}, i=1,2, \ldots N, \\
\phi_{i}^{\text {local }}: i \text {-th node local fluxes, } \\
M_{i}^{\text {local }}: i \text {-th node local system matrix, } \\
S_{i}^{\text {local }}: i \text {-th node local fixed source. }
\end{gathered}
$$

In this HCMFD framework, the whole-core pin-by-pin analysis can be performed very effectively by an efficient parallel computing in a local-global non-linear iterative scheme as shown in Fig. 1. A whole-core pin-by-pin neutronics calculation for a large PWR core only takes 13.7 seconds with 40 cores on Intel Xeon Gold 6148 CPUs $(2.40 \mathrm{GHz})$. More details of the work can be found in the reference [1].

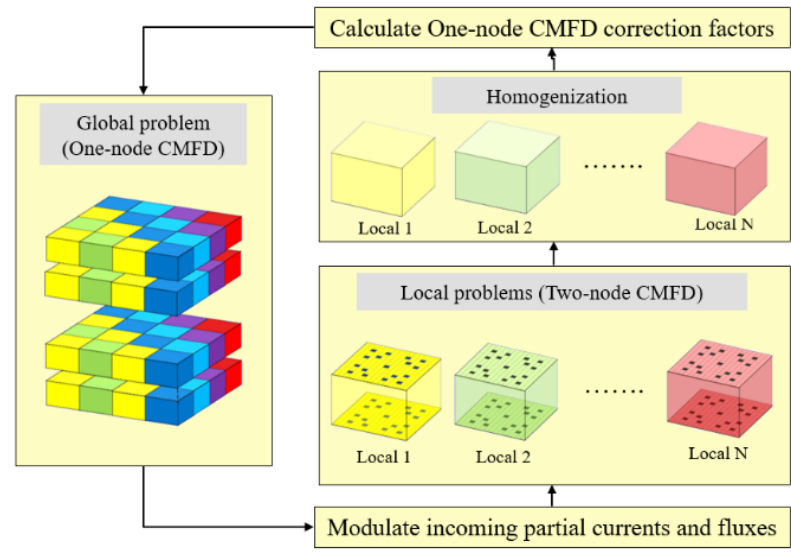

Figure 1. Schematic diagram of the HCMFD algorithm 


\subsection{GPS Method}

The GPS method is a combination of the generalized equivalence theory (GET) [5] and the superhomogenization method (SPH) [6] to reduce the error in the pin-wise group constants. In the GPS method, the pin-wise and XS-wise SPH factor concept is introduced to correct the standard GET-based pin-wise XSs. To reflect the neighborhood effects in the GPS correction, the SPH factors are functionalized by the pin-wise current-to-flux ratio (CFR), as defined by Eqs. (2) and (3) for 2-D and 3-D geometries, respectively. These CFR value is a kind of quantitative expression of the neighborhood effect. The nodewise flux and surface-wise net currents are appropriately weighted by area and length or by volume and area so that the two definitions for each dimension are equivalent and compatible.

$$
\begin{aligned}
C F R_{g}^{2 D}=\frac{\sum_{S} J_{g}^{S} \cdot l^{S}}{\phi_{g} \cdot A} & =\frac{\sum_{S} J_{g}^{S} \cdot l^{S}}{\phi_{g} \cdot A} \times \frac{h}{h}=\frac{\sum_{\text {radial } S} J_{g}^{S} \cdot A^{S}}{\phi_{g} \cdot V} \\
C F R_{g}^{3 D} & =C F R_{g}^{2 D}+\frac{\sum_{\text {axial } S} J_{g}^{S} \cdot A^{S}}{\phi_{g} \cdot V} \\
l & =\text { Node radial pitch, } \\
A & =\text { 2-D node area, } \\
h & =\text { Node axial height, } \\
V & =\text { 3-D node volume. }
\end{aligned}
$$

In the GPS method, the XS correction is performed using the pin-wise GPS functions that are predetermined for each assembly type. The GPS functions are of pin-wise CFR values, and the CFR values for the correction are obtained by the initial steady-state analysis without any correction. After the XS update, another steady-state calculation is performed with the corrected XSs. Since one steady-state analysis takes a short time by the HCMFD algorithm, such a two-step GPS correction can be cost-effective. The overall flow of the GPS correction is shown in Fig. 2.

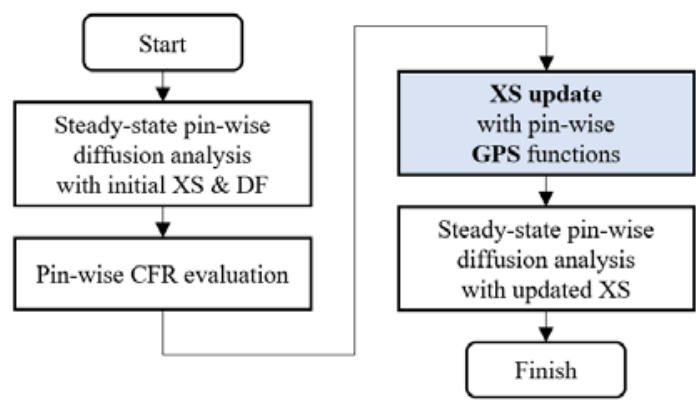

Figure 2. Flowchart of the GPS method

The pin-wise and XS-wise SPH factors of the GPS method are evaluated by using a set of color-set models, as described in Fig. 3, with randomly-arranged assemblies, so that various possible neighboring conditions are taken into account. A color-set calculation consists of a reference transport calculation with a heterogeneous geometry, and repeated nodal diffusion color-set calculations with pin-wisely homogenized group constants. The pin-wise SPH factors for each assembly type that satisfy Eq. (4) are obtained by the color-set SPH iteration of repeated pin-wise diffusion calculations and XS updates as shown in Fig. 4. Then, the converged SPH factors and the CFR values of the nodal diffusion color-set solutions for each color-set model are used as raw fitting data set for the GPS function generation. 


$$
\begin{aligned}
& \tau_{g, x}^{\text {color-set }}=S P H_{g, x} \sum_{g, x}^{S A} \phi_{g, \text { dif }}^{\text {color }- \text { set }} \\
& g \quad=\text { Group index, } \\
& x=\text { Reaction type index, } \\
& \tau_{g, x}^{\text {color-set }}=\text { Reference pin-wise reaction rate by transport calc., } \\
& \phi_{g, d i f}^{\text {color-set }}=\text { Pin-wise flux by nodal diffusion calc., } \\
& \Sigma_{g, x}^{S A} \quad=\text { Pin-wise XS by single-assembly tranport calc., } \\
& \mathrm{SPH}_{g, x}=\mathrm{XS} \text {-wise } \mathrm{SPH} \text { factor. }
\end{aligned}
$$

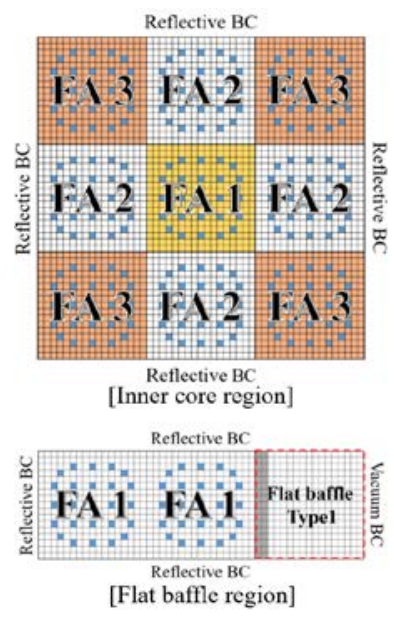

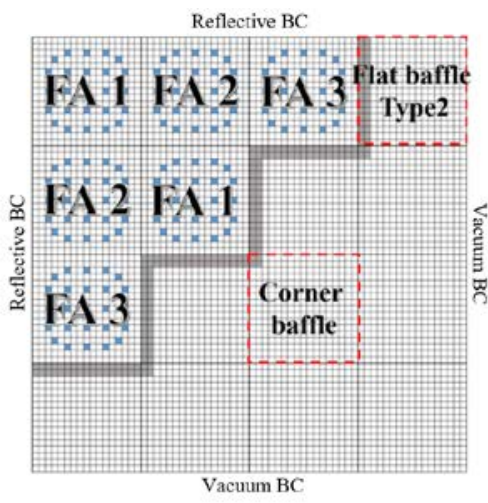

[Baffle-reflector region, type 1]

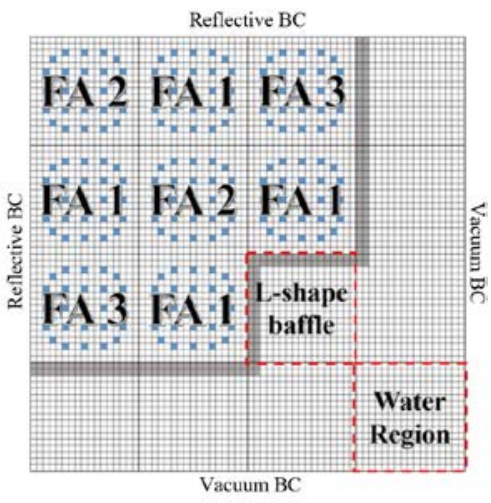

[Baffle-reflector region, type 2]

Figure 3. Color-set models for GPS function generation

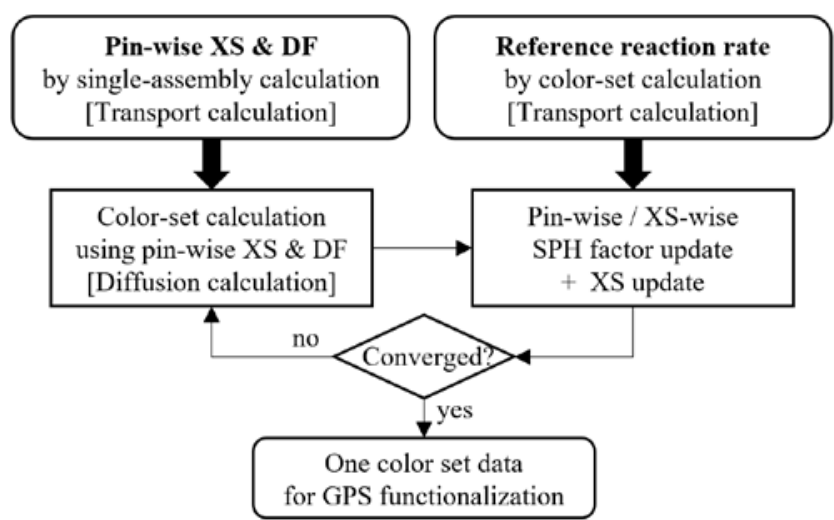

Figure 4. Data generation for GPS function fitting (color set calculation)

In the GPS method, the changes in the SPH factors are functionalized by the changes in the two-group CFR values. As specified in Eqs. (5) and (6), the changes mean the differences of those values in each reference color-set calculation and the single-assembly lattice calculation. The SPH factors in the lattice calculation are considered to be 1.0, since the pin-wise DFs generated by the lattice calculation are used in the colorset diffusion calculation. The pin-wise DFs are to preserve the heterogeneous quantities in a lattice geometry, and it means that one does not need any further XS correction using non-unity SPH factors.

$$
\begin{aligned}
\Delta S P H_{g, x} & =S P H_{g, x}^{\text {color-set }}-S P H_{g, x}^{S A} \\
\Delta C F R_{g} & =C F R_{g}^{\text {color-set }}-C F R_{g}^{S A}
\end{aligned}
$$

The detailed GPS functions are expressed in Eqs. (7) and (8), which were determined in the previous studies. It is worthwhile to note that the GPS functions are based on bilinear correlations. The coefficients for both 
group delta CFR values and a constant term are obtained by the color-set fitting data sets. With the predefined pin-wise and XS-wise GPS functions for each assembly type, the XSs are updated using the pinwise CFR information.

$$
\begin{aligned}
& \Delta S P H_{F, x}=a_{1, F, x} \Delta C F R_{F, x}+a_{2, F, x} \Delta C F R_{\mathrm{T}, x}+a_{3, F, x} \\
& \Delta S P H_{T, x}=a_{1, T, x} \Delta C F R_{F, x}+a_{2, T, x} \Delta C F R_{\mathrm{T}, x}+a_{3, T, x} \\
& \Sigma_{g, x}^{\text {corrected }}=\left(1+\Delta S P H_{g, x}\right) \cdot \Sigma_{g, x}^{S A}
\end{aligned}
$$

Previously, the GPS functions generated by 2-D color-set calculations have been used only in 2-D benchmark problems. In this work, the feasibility of adapting the 2-D GPS functions in several 3-D benchmark problems is scrutinized.

\section{NUMERICAL RESULTS}

A 2-D method of characteristics (MOC) based lattice code, DeCART2D [7] was used for the lattice calculations and color-set calculations for the GPS functions. The 3-D pin-by-pin diffusion analyses were performed by an in-house HCMFD code. The reference core calculations were performed by a continuous energy Monte Carlo (MC) code, Serpent2 [8] with the ENDF/B-VII.1 library.

It should be mentioned that the computer codes for the reference solution and the GPS functionalization, Serpent2 and DeCART2D respectively, are different. Thus, the error between the corrected diffusion solution and the reference MC solution should contain the error between those two codes. In the near future, the GPS functions will be also determined by Serpent 2 for consistency.

Figure 5 shows the fuel assembly geometry and 3 core configurations are given in Fig. 6. The detailed 3-D quarter core geometry is described in Table I. The axial DFs are only considered on the interface between the axial reflector layers and fuel assemblies. In the diffusion calculations, the axial mesh size was fixed to $20 \mathrm{~cm}$ in both local and global problems. This study is focused only on the accuracy of the solution since the computing time for such a small-size problem is so short that the parallel efficiency cannot be meaningfully measured.

Table I. Parameters for the 3-D quarter core geometry

\begin{tabular}{|c|c|c|}
\hline \multirow{2}{*}{ Radial configuration } & No. of assemblies & 13 \\
\cline { 2 - 3 } & No. of baffle/reflector nodes & 12 \\
\hline \multirow{2}{*}{ Axial configuration } & Top \& bottom reflector layer & $20 \mathrm{~cm} \times 1$ \\
\cline { 2 - 3 } & Active core layers & $20 \mathrm{~cm} \times 10$ \\
\hline \multicolumn{2}{|c|}{ Assembly geometry } & $21.42 \mathrm{~cm} \times 21.42 \mathrm{~cm}(17$ by 17 pins $)$ \\
\hline
\end{tabular}

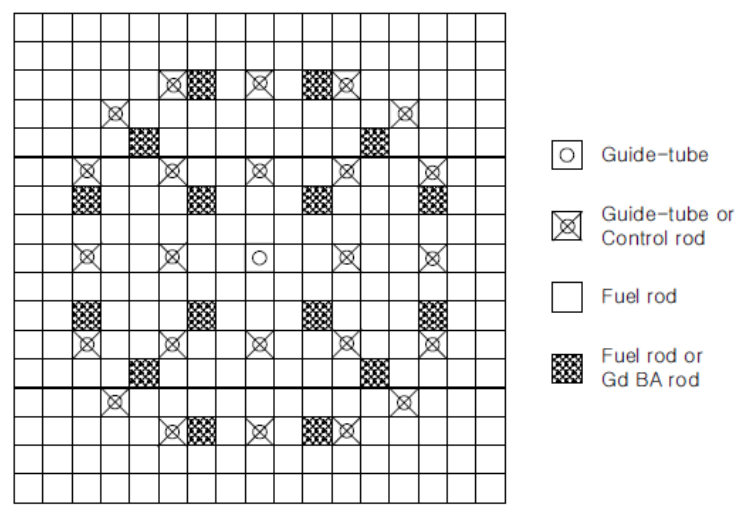

Figure 5. UOX fuel assembly geometry in the KAIST-1A benchmark [9] 


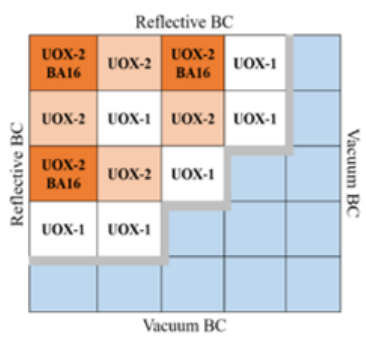

[Problem 1]

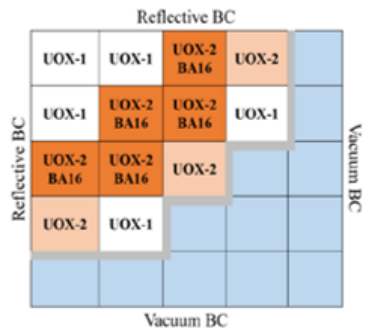

[Problem 3]

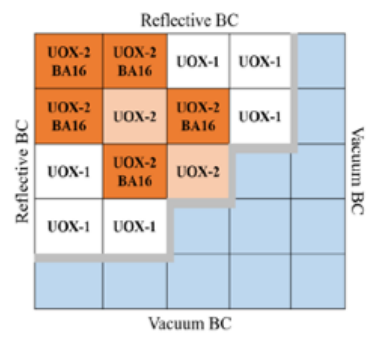

[Problem 2]

UOX-1: (U235: 2.0w/0, U238: 98.0w/o) UOX-2: (U235: 3.3w/o, U238: $96.7 \mathrm{w} / 0$ ) BA16 : 16 Gd BA rods inserted $-\mathrm{UO}_{2}:(0.711 \mathrm{w} / \mathrm{o} \mathrm{U235})+\mathrm{Gd}_{2} \mathrm{O}_{3}(9.0 \mathrm{w} / \mathrm{o})$

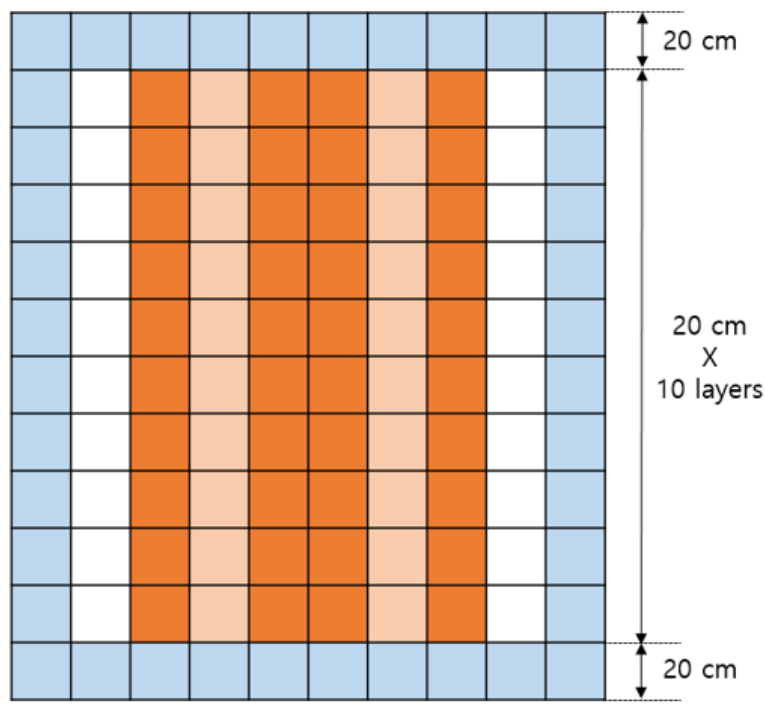

[Axial Configuration]

Figure 6. Radial and axial core configurations in the 3 benchmarks

Prior to the application of 2-D GPS functions to the 3-D problems, effectiveness of the 2-D GPS functions in 2-D geometry are summarized in Table II. The effects of the same GPS functions in the 3-D problems were then evaluated and given in Table III in terms of k-eff. The results without the GPS correction correspond to the initial steady-state results, the standard two-step calculation. It could be noticed that the performance of the 2-D GPS functions in 3-D problems are rather comparable to that of 2-D geometries.

Table II. Effects of 2-D GPS correction in 2-D geometries

\begin{tabular}{|c|c|c|c|c|c|c|}
\hline \multirow{2}{*}{ Prob. \# } & \multicolumn{2}{|c|}{$k_{\text {eff }}$ error } & \multicolumn{2}{c|}{ Pin power RMS error (\%) } & \multicolumn{2}{c|}{ Pin power maximum error (\%) } \\
\cline { 2 - 7 } & w/o GPS & with GPS & w/o GPS & with GPS & w/o GPS & with GPS \\
\hline 1 & 85.22 & 22.54 & 0.82 & 0.21 & 2.95 & 1.64 \\
\hline 2 & 56.88 & 21.32 & 0.77 & 0.26 & 2.75 & 1.30 \\
\hline 3 & 27.97 & 11.79 & 0.82 & 0.28 & 2.76 & 1.20 \\
\hline
\end{tabular}

Table III. Comparison of k-eff for 3-D problems

\begin{tabular}{|c|c|c|c|c|c|c|}
\hline \multirow{2}{*}{ Type } & \multicolumn{2}{|c|}{ Problem 1 } & \multicolumn{2}{c|}{ Problem 2 } & \multicolumn{2}{c|}{ Problem 3 } \\
\cline { 2 - 7 } & $\mathrm{k}_{\text {eff }}$ & Error $(\mathrm{pcm})$ & $\mathrm{k}_{\text {eff }}$ & Error $(\mathrm{pcm})$ & $\mathrm{k}_{\text {eff }}$ & Error $(\mathrm{pcm})$ \\
\hline Ref. (Serpent2) & 1.099221 & - & 1.041678 & - & 1.042212 & - \\
\hline w/o GPS & 1.100301 & +107.99 & 1.042670 & +99.21 & 1.042987 & +77.55 \\
\hline with GPS & 1.099498 & +27.68 & 1.042155 & +47.79 & 1.042655 & +44.34 \\
\hline
\end{tabular}

To analyze the results in more detail, the pin power errors at each layer were investigated and are shown in Tables IV to VI. Basically, the overall pin power error gets larger in 3-D problems due to an increase in the pin power peaking factor compared to 2-D problems. Such error can be easily amplified for pins having very small level of power, which is clearly demonstrated in the maximum pin power error of each layer. The maximum errors exceed 30\% at the top layers in Problem 2 and 3, where such layers contain the lowest pin power. It is considered that the XSs and DFs of the axial reflector layers, which were generated from 2$\mathrm{D}$ geometry, may have not aptly reflected the problem and contributed as an additional error.

Nevertheless, there was noticeable reduction in the pin power RMS errors at the vicinity of central layers, after the implementation of the GPS corrections. It indicates the 2-D GPS functions can adjust the XSs 
properly for such regions since the axial neutron leakages are sufficiently small at the central layers. However, the GPS corrections are rather unnoticeable at the boundary layers due to the 2-D GPS functions and inconsistency between the DeCART2D and reference Serpent methods.

The radial pin power error distributions in Problem 2 at layer 5 are plotted in Fig. 7, which clearly depicts the reduction in overall pin power error after the GPS correction. The red dots are the fuel pins including the burnable absorber, and it was ascertained that such a large error has been caused by the innate disparity between DeCART2D and Serpent2. The small increase in the pin power maximum error at Layers 2-9 of Table IV to VI are also due to the presence of such burnable absorber pins.

Table IV. Layer-wise pin power error (Problem 1)

\begin{tabular}{|c|c|c|c|c|}
\hline \multirow{2}{*}{ Layer \# } & \multicolumn{2}{|c|}{ Pin power RMS error (\%) } & \multicolumn{2}{c|}{ Pin power maximum error (\%) } \\
\cline { 2 - 5 } & w/o GPS & with GPS & w/o GPS & with GPS \\
\hline 10 (Top) & 2.41 & 2.62 & 22.74 & 14.31 \\
9 & 1.91 & 1.49 & 6.85 & 7.82 \\
8 & 1.72 & 1.25 & 6.53 & 7.40 \\
7 & 1.63 & 1.17 & 6.40 & 7.21 \\
6 & 1.56 & 1.09 & 6.26 & 6.91 \\
5 & 1.56 & 1.08 & 6.36 & 6.92 \\
4 & 1.54 & 1.10 & 6.28 & 6.69 \\
3 & 1.64 & 1.19 & 6.29 & 7.13 \\
2 & 1.74 & 1.30 & 6.72 & 7.55 \\
1 (Bottom) & 1.87 & 2.04 & 11.71 & 9.49 \\
\hline Total & 1.78 & 1.51 & 22.74 & 14.31 \\
\hline
\end{tabular}

Table V. Layer-wise pin power error (Problem 2)

\begin{tabular}{|c|c|c|c|c|}
\hline \multirow{2}{*}{ Layer \# } & \multicolumn{2}{|c|}{ Pin power RMS error (\%) } & \multicolumn{2}{c|}{ Pin power maximum error (\%) } \\
\cline { 2 - 5 } & w/o GPS & with GPS & w/o GPS & with GPS \\
\hline 10 (Top) & 3.06 & 2.60 & 38.11 & 24.67 \\
9 & 1.76 & 1.28 & 7.05 & 8.06 \\
8 & 1.50 & 1.06 & 6.21 & 7.03 \\
7 & 1.48 & 1.07 & 6.49 & 7.25 \\
6 & 1.47 & 1.08 & 5.95 & 6.74 \\
5 & 1.47 & 1.09 & 5.98 & 6.70 \\
4 & 1.51 & 1.06 & 6.05 & 6.71 \\
3 & 1.56 & 1.07 & 6.11 & 6.88 \\
2 & 1.70 & 1.19 & 6.70 & 7.55 \\
1 (Bottom) & 2.10 & 1.99 & 20.84 & 12.95 \\
\hline Total & 1.82 & 1.44 & 38.11 & 24.67 \\
\hline
\end{tabular}

Table VI. Layer-wise pin power error (Problem 3)

\begin{tabular}{|c|c|c|c|c|}
\hline \multirow{2}{*}{ Layer \# } & \multicolumn{2}{|c|}{ Pin power RMS error (\%) } & \multicolumn{2}{|c|}{ Pin power maximum error (\%) } \\
\cline { 2 - 5 } & w/o GPS & with GPS & w/o GPS & with GPS \\
\hline 10 (Top) & 3.63 & 3.01 & 37.13 & 23.36 \\
9 & 2.29 & 1.51 & 7.59 & 8.29 \\
8 & 2.02 & 1.20 & 6.42 & 7.33 \\
7 & 1.82 & 1.07 & 6.15 & 6.52 \\
6 & 1.78 & 1.07 & 6.12 & 6.99 \\
5 & 1.77 & 1.07 & 5.93 & 6.84 \\
4 & 1.83 & 1.10 & 5.62 & 6.64 \\
3 & 1.87 & 1.13 & 6.94 & 7.99 \\
2 & 2.01 & 1.22 & 6.61 & 7.63 \\
1 (Bottom) & 2.53 & 2.10 & 21.29 & 12.20 \\
\hline Total & 2.22 & 1.57 & 37.13 & 23.36 \\
\hline
\end{tabular}




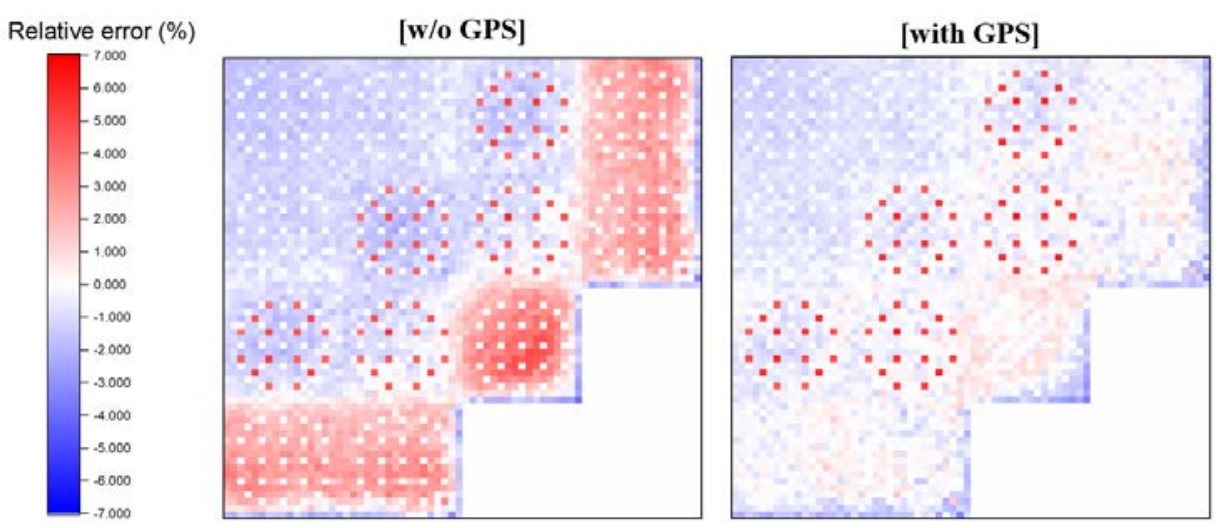

Figure 7. Radial pin power error at layer 5 (Problem 2)

\section{CONCLUSIONS}

It was demonstrated that the GPS functions generated by 2-D color-set calculations can also be utilized in 3-D geometries that consist of the same assembly types without any critical problems. Currently, the effectiveness of the application of 2-D GPS functions in 3-D problems is limitedly verified at the internal layers where the axial neutron leakage is relatively small. The results will be reproduced soon with the consistency between the color-set calculation and reference calculation using Serpent2. Also, the 3-D GPS functions generated by 3 -D color-set models will be considered.

\section{ACKNOWLEDGMENTS}

This work was supported by the National Research Foundation of Korea (NRF) Grant funded by the Korean Government (MSIP) (NRF-2016R1A5A1013919).

\section{REFERENCES}

1. J. Kim and Y. Kim, "Three-Dimensional Pin-Resolved Transient Diffusion Analysis of PWR Core by the Hybrid Coarse-Mesh Finite Difference Algorithm," Nuclear Science and Engineering, Published online, 09 Aug (2019).

2. H. Yu, J. Kim, and Y. Kim, "Pinwise Diffusion Solution of Partially MOX-Loaded PWRs with the GPS (GET PLUS SPH) Method," Nuclear Science and Engineering, Published online, 10 June (2019).

3. H. C. Shin and Y. Kim, "A Nonlinear Combination of CMFD (Coarse-Mesh Finite Difference) and FMFD (Fine-Mesh Finite Difference) Methods," Proc. Korean Nuclear Society Spring Mtg., Pohang, Korea, May (1999).

4. K.S. Smith, "Nodal method storage reduction by nonlinear iteration," Trans. Am. Nucl. Soc., 44, p.265 (1983).

5. K. S. Smith, “Assembly Homogenization Techniques for Light Water Reactor Analysis," Progress in Nuclear Energy, 17, 303 (1986).

6. A. Hebert, "A Consistent Technique for the Pin-by-Pin Homogenization of a Pressurized Water Reactor Assembly", Nucl. Sci. Eng., 113, 227 (1993).

7. "DeCART 2D v1.0 User's Manual," KAERI/TR-5116/2013, Korea Atomic Energy Research Institute (2013).

8. J. Leppanen, "PSG2 / Serpent - a Continuous-energy Monte Carlo Reactor Physics Burnup Calculation Code," VTT Technical Research Centre of Finland, 13 June (2012).

9. N. Z. Cho, "Benchmark Problem 1A: MOX Fuel-Loaded Small PWR Core (MOX Fuel with Zoning)", KAIST/NuratT, 23 June (2000). 